\title{
A plant-specific DYRK kinase DYRKP coordinates cell morphology in Marchantia polymorpha
}

\author{
Tomoyuki Furuya $^{1,2} \cdot$ Haruka Shinkawa $^{3,4} \cdot$ Masataka Kajikawa $^{3,5} \cdot$ Ryuichi Nishihama $^{3,6} \cdot$ Takayuki Kohchi $^{3}$. \\ Hideya Fukuzawa ${ }^{3} \cdot$ Hirokazu Tsukaya $^{1}[$
}

Received: 16 June 2021 / Accepted: 1 September 2021 / Published online: 21 September 2021

(c) The Author(s) 2021

\begin{abstract}
Dual-specificity tyrosine phosphorylation-regulated kinases (DYRKs) are activated via the auto-phosphorylation of conserved tyrosine residues in their activation loop during protein translation, and they then phosphorylate serine/threonine residues on substrates. The DYRK family is widely conserved in eukaryotes and is composed of six subgroups. In plant lineages, DYRK homologs are classified into four subgroups, DYRK2s, yet another kinase1s, pre-mRNA processing factor 4 kinases, and DYRKPs. Only the DYRKP subgroup is plant-specific and has been identified in a wide array of plant lineages, including land plants and green algae. It has been suggested that in Arabidopsis thaliana DYRKPs are involved in the regulation of centripetal nuclear positioning induced by dark light conditions. However, the molecular functions, such as kinase activity and the developmental and physiological roles of DYRKPs are poorly understood. Here, we focused on a sole DYRKP ortholog in the model bryophyte, Marchantia polymorpha, MpDYRKP. MpDYRKP has a highly conserved kinase domain located in the C-terminal region and shares common sequence motifs in the N-terminal region with other DYRKP members. To identify the roles of MpDYRKP in M. polymorpha, we generated loss-of-function Mpdyrkp mutants via genome editing. Mpdyrkp mutants exhibited abnormal, shrunken morphologies with less flattening in their vegetative plant bodies, thalli, and male reproductive organs, antheridial receptacles. The surfaces of the thalli in the Mpdyrkp mutants appeared uneven and disordered. Moreover, their epidermal cells were drastically altered to a narrower shape when compared to the wild type. These results suggest that MpDYRKP acts as a morphological regulator, which contributes to orderly tissue morphogenesis via the regulation of cell shape.
\end{abstract}

Keywords Cell shape $\cdot$ Liverwort $\cdot$ Morphogenesis $\cdot$ Protein kinase

Hirokazu Tsukaya

tsukaya@bs.s.u-tokyo.ac.jp

1 Graduate School of Science, The University of Tokyo, Tokyo 113- 0033, Japan

2 Graduate School of Science, Kobe University, Kobe 657-8501, Japan

3 Graduate School of Biostudies, Kyoto University, Kyoto 606-8502, Japan

4 Present Address: Research Institute for Bioresources and Biotechnology, Ishikawa Prefectural University, Ishikawa 921-8836, Japan

5 Present Address: Faculty of Biology-Oriented Science and Technology, Kindai University, Wakayama 649-6493, Japan

6 Present Address: Faculty of Science and Technology, Tokyo University of Science, Chiba 278- 8510, Japan

\section{Introduction}

The dual-specificity tyrosine (Y) phosphorylation-regulated kinase (DYRK) family belongs to the larger Cdk2, MAPK, GSK3, CLK and related kinase family (CMGC), that has been identified in a wide range of animals, plants, fungi, and protists (Aranda et al. 2011; Manning et al. 2002; Varjosalo et al. 2013). Generally, DYRKs are activated via the autophosphorylation of a conserved $\mathrm{Y}$ residue in the activation loop during protein translation and they then phosphorylate serine/threonine $(\mathrm{S} / \mathrm{T})$ residues on substrates. (Lochhead et al. 2005; Soppa and Becker 2015). The DYRK family is composed of six subgroups: DYRK1s, DYRK2s, yet another kinase1s (Yak1s), pre-mRNA processing factor 4 kinases (PRP4Ks), homeodomain-interacting protein kinases (HIPKs), and plant-specific DYRKs (DYRKPs) (Kajikawa et al. 2015). DYRKs in yeast and mammals are well known 
as key regulators of the cell cycle and differentiation (Aranda et al. 2011; Becker 2012; Soppa and Becker 2015). Yak1 in Saccharomyces cerevisiae, which is a founding member of the DYRK family, is a negative regulator of proliferation under nutritional stress (Garret and Broach 1989). Mammalian DYRK1A, which is well known as a candidate gene for involvement in Down syndrome, functions in neuronal differentiation (Gwack et al. 2006; Kurabayashi and Sanada 2013). In addition, DYRK2 plays important roles in cell cycle and apoptosis regulation (Nihira and Yoshida 2015; Taira et al. 2007, 2012).

There are only four DYRK subgroups in plant lineages, including land plants and green algae, and these include DYRK2, Yak1, PRP4K, and DYRKP. Triacylglycerol accumulation regulator1 (TAR1), a homolog of the Yak subgroup in Chlamydomonas reinhardtii, was identified as the gene responsible for the low triacylglycerol (TAG) accumulation mutant that is known to occur in sulfur and nitrogen deficient conditions (Kajikawa et al. 2015). Under nitrogen-deficient conditions, TAR1 also regulates the growth and degradation of chlorophyll and photosynthesis-related proteins. The lossof-function mutant for Arabidopsis thaliana Yak homolog, AtYAK1, showed less sensitivity to the plant hormone abscisic acid (ABA) in the context of seed germination, seedling growth, and stomatal closure, suggesting that AtYAK1 positively regulates the ABA signaling transduction pathway (Kim et al. 2016). In addition, AtYAK1 was also found to interact with LIGHT-REGULATED WD1 (LWD1) and LWD2, which are key regulators of the circadian period (Huang et al. 2017). Indeed, atyakl mutants have a longer circadian period length and delayed flowering phenotype than the wild type (Huang et al. 2017). Moreover, AtYAK1 functions downstream of TARGET OF RAPAMAYCIN (TOR) kinase, which is involved in the regulation of meristem activity and cell differentiation during root development (Barrada et al. 2019; Forzani et al. 2019; Xiong et al. 2013).

Unlike the other subgroups, the DYRKP subgroup is only found in plant genomes (Kajikawa et al. 2015; Schulz-Raffelt et al. 2016). In A. thaliana, four DYRKPs (DYRKP-1, DYRKP-2 A, DYRKP-2B, and DYRKP-3) are encoded in the genome (Iwabuchi et al. 2019). These DYRKPs were shown to interact with ANGSTIFOLIA (AN) in the yeast two-hybrid system, and it is suggested that this complex contributes to dark-induced centripetal nuclear positioning via the alignment of actin filaments (Iwabuchi et al. 2019). In addition, DYRKP in $C$. reinhardtii negatively regulates the accumulation of starch and oil synthesis in low energy status conditions (Schulz-Raffelt et al. 2016). However, the functions of DYRKPs in other contexts, including their morphological, developmental, and evolutionary aspects, requires further elucidation.

Here, we focused on the liverwort Marchantia polymorpha, which is part of the bryophyte (Bowman et al. 2017).
M. polymorpha is a well-established model plant with various experimental advantages such as a short life cycle, ease of culturing and crossing, a low level of genetic redundancy, and available genetic information and gene manipulation systems (Ishizaki et al. 2016; Kohchi et al. 2021; Shimamura 2016). Indeed, M. polymorpha has a single DYRKP ortholog, MpDYRKP. We generated genome-edited mutants, Mpdyrkp, which exhibited defects in their thallus morphology as they showed shrinking and less flattening. Moreover, epidermal cells in Mpdyrkp mutants were drastically altered to narrower shapes compared to the wild type. Our findings provide novel knowledge regarding the role of DYRKPs in terms of morphology.

\section{Materials and methods}

\section{Plant materials and culture conditions}

The M. polymorpha male accession Takaragaike-1 (Tak-1) was used as the wild-type line (Ishizaki et al. 2008; Okada et al. 2000). Wild-type and transgenic plants were cultured on half-strength Gamborg's B5 medium (Gamborg et al. $1968)$ containing $1 \%(\mathrm{w} / \mathrm{v})$ agar with or without $1 \%(\mathrm{w} / \mathrm{v})$ sucrose or soil under $50-60 \mu \mathrm{mol} \mathrm{m} \mathrm{m}^{-2} \mathrm{~s}^{-1}$ continuous white light at $22{ }^{\circ} \mathrm{C}$. To induce the reproductive phase, gemmalings were cultured on soil under continuous 50-60 $\mu \mathrm{mol}$ $\mathrm{m}^{-2} \mathrm{~s}^{-1}$ white light supplemented with $20-40 \mu \mathrm{mol} \mathrm{m} \mathrm{m}^{-2} \mathrm{~s}^{-1}$ far-red light $(730 \mathrm{~nm})$ irradiation (VBL-T600-1, Valore).

\section{Construction of transgenic plants}

The MpDYRKP targeting vectors used for genome editing with the CRISPR/Cas9 system were constructed according to Sugano et al. (2018). MpDYRKP-gRNAF1 5'-CTC GGT CCT ATT TTC AGG GGC A-3' and MpDYRKP-gRNA-R1 5'-AAA CTG CCC CTG AAA ATA GGA C-3' for guide RNA-1, and MpDYRKP-gRNAF2 5'-CTC GAT CGT ACA CAG GAA AAA C-3' and MpDYRKP-gRNA-R2 5'-AAA CGT TTT TCC TGT GTA CGA T-3' for guide RNA-2 were incubated together at $95^{\circ} \mathrm{C}$ for $5 \mathrm{~min}$, then cooled to $85^{\circ} \mathrm{C}$ at a rate of $-2{ }^{\circ} \mathrm{C}$ $\mathrm{s}^{-1}$, held at $85^{\circ} \mathrm{C}$ for $1 \mathrm{~min}$, the further cooled to $25^{\circ} \mathrm{C}$ at $-0.2{ }^{\circ} \mathrm{C} \mathrm{s}^{-1}$ to enable annealing. The resultant annealed oligonucleotides containing the target sequence were inserted into the $B s a \mathrm{I}$ site of pMpGE_En03 to construct the entry vector. The sequence between attL1 and attL2 within the entry vector was inserted into the binary vector pMpGE010 by the LR reaction using LR clonase II (Life Technologies). The vector used for the complementation analysis was constructed based on the method of Ishizaki et al. (2015). The genomic sequences of the promoter region (5-kb upstream of the start codon) and 
terminator region (1-kb downstream of the stop codon) of $\mathrm{MpDYRKP}$ were amplified from the Tak-1 genomic DNA using the following primers: MpDYRKPpro-F 5'-TGG ATC CGG TAC CGA ATT CGG ACT TGG AAC ATC TAG C-3' and MpDYRKPpro-R, 5'-CGA GTG CGG CCG CGA ATT GGC TGG ATA TGA TTG AGA TCA C-3' for the promoter, and MpDYRKPterm-NotI-F 5'-TAT CTC CGC GCG GCC GTG AGA GTT ATC TTT CAA TC-3' and MpDYRKPterm-NotI-R1 5'-ATC TCG AGT GCG GCC TGT GGT TTA CCA TAT AAT TC-3" for the terminator. The stop codon in the MpDYRKPterm-NotI-F primer is underlined in the above sequence. The longest MpDYRKP coding sequence corresponding to the splicing variant Mp3g19940.1, was amplified from Tak-1 thalli cDNA using the following primers: MpDYRKPORF-NotIF 5'-ATC CAG CCA ATT CGC GGC CAT GGC GGA TTC GGT CAA TGC C-3' and MpDYRKPORF-NotI-R 5'-AGA TAT CTC GAG TGC GGC CGC GCG GAG ATA GGT TCA TAT GG-3'. The MpDYRKP promoter region (hereafter ${ }_{p r o} \mathrm{MpDYRKP}$ ) was cloned into pENTR1A digested with EcoRI using the SLiCE method (Motohashi 2015). Then, the MpDYRKP ORF was inserted into the NotI site of pENTR1A- ${ }_{p r o} \mathrm{MpDRYKP}$. During this cloning, the Not 1 site on the $3^{\prime}$ side of the ORF was preserved, while the Not 1 site on the $5^{\prime}$ side of the ORF was not. Finally, the MpDYRKP terminator region (hereafter MpDYRKPterm) was inserted into the NotI site of the pENTR1A- ${ }_{p r o} \mathrm{Mp} D Y R K P: \mathrm{Mp} D Y R K P$ ORF plasmid. The ${ }_{\text {pro }} \mathrm{Mp} D Y R K P: \mathrm{Mp} D Y R K P$ ORF:MpDYRKPterm region between attL1 and attL2 within the resultant entry plasmid was inserted into the destination vector pMpGWB301 (Ishizaki et al. 2015) by LR reaction using LR clonase II to generate the ${ }_{p r o} \mathrm{MpDYRKP:MpDYRKP}$ construct. To confirm the introduction of the ${ }_{\text {pro }} \mathrm{MpDYRKP:MpDYRKP}$ construct in the transformants, the primers, MpDYRKPORFE2-F1 5'-GCA AGG AGG CTT AGT GGA GGT A-3' and MpDYRKPORFE2-R1 5'-TCT CCA CTT CCT CGT CAT CAT C-3' were used for PCR screening.

A citrine fusion construct, pro $\mathrm{Mp} D Y R K P: \mathrm{Mp} D Y R K P$ Citrine was constructed as previously reported (Ishizaki et al. 2015). The constructed entry plasmid pENTR1A${ }_{\text {pro }} \mathrm{MpDYRKP:MpDYRKP}$ ORF, which did not have a stop codon, was used for LR cloning with the destination vector pMpGWB307 (Ishizaki et al. 2015).

The thallus transformation with the CRISPR/Cas9 system and complementation constructs were described previously (Kubota et al. 2013). Thalli from Mpdyrkp$1^{g e}$ and Agrobacterium containing the pMpGWB301-

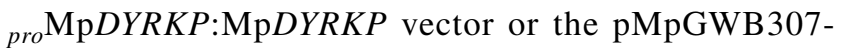

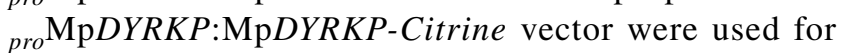
the transformation. Transformants were selected on halfstrength B5 agar medium containing $1 \%$ (w/v) agar, 0.5 $\mu \mathrm{M}$ chlorosulfuron, and $100 \mathrm{mg} \mathrm{L}^{-1}$ cefotaxime (Claforan; Sanofi-Aventis).

\section{Phylogenetic analysis}

A phylogenetic tree for the DYRKs was constructed by comparing the amino acid sequences of their kinase domains. The tree was generated using MEGA X (version 10.1, https://www.megasoftware.net/; Kumar et al. 2018) with the maximum likelihood algorithm.

\section{Confocal microscopy}

For the observation of apical notches, 3-day-old plants after gemma germination were fixed in PFA fixative solution [ $4 \%$ (w/v) paraformaldehyde, and $0.05 \%$ (v/v) Triton X-100 in Phosphate buffered saline (PBS)]. Samples were degassed by vacuum infiltration and incubated for $1 \mathrm{~h}$ at room temperature with gentle shaking. Fixed samples were rinsed with $0.05 \%$ (v/v) Triton X-100 in PBS and cleared in the ClearSee solution $[10 \%(\mathrm{w} / \mathrm{v})$ xylitol, $15 \%(\mathrm{w} / \mathrm{v})$ sodium deoxycholate, and $25 \%(\mathrm{w} / \mathrm{v})$ urea] with $0.02 \%(\mathrm{v} / \mathrm{v})$ SCRI Renaissance 2200 (Renaissance Chemicals, Selby, UK) (Kurihara et al. 2015). After replacing with the ClearSee solution, samples were kept for two additional days or more. Samples were mounted on glass slides with the ClearSee solution for microscopic obervations. SCRI Renaissance 2200 fluorescence was visualized using the confocal laser scanning microscope (Olympus FLUOVIEW FV1000, Tokyo, Japan) at excitation and detection wavelengths of 405 and 425-460 nm, respectively. Images of plant tissues expressing the MpDYRKP-Citrine fusion protein were captured using a Leica TCS SP8 microscope (Leica Microsystems). A 488$\mathrm{nm}$ argon laser was used for excitation, and fluorescence detected between 500 and $550 \mathrm{~nm}$ and 705-765 nm was from Citrine and chlorophyll, respectively.

\section{5-Ethynyl-2'-deoxyuridine (EdU) staining}

To visualize S-phase cells, the Click-iT EdU Imaging Kit (Life Technologies) was used. EdU staining was performed as described previously (Furuya et al. 2018; Naramoto et al. 2019; Nishihama et al. 2015), with a slight modification. Three-day-old plants after gemma germination were soaked in half-strength Gamborg's B5 medium with $10 \mu \mathrm{M}$ EdU at $22{ }^{\circ} \mathrm{C}$ for $1 \mathrm{~h}$ under continuous white light. Thalli were fixed in FAA [50\% (v/v) ethyl alcohol, $2.5 \%(\mathrm{w} / \mathrm{v})$ glacial acetic acid, and $2.5 \%(\mathrm{w} / \mathrm{v})$ formalin] and were degassed by vacuum infiltration. EdU in the fixed samples were detected with Alexa Fluor 555-azide following the manufacturer's protocol. For clearing, samples were treated with the ClearSee solution for a few days. Samples were mounted on glass slides with the ClearSee solution; Alexa Fluor555 
fluorescence was visualized using the confocal laser scanning microscope (Olympus FLUOVIEW FV1000, Tokyo, Japan) at excitation and detection wavelengths of 559 and $570-670 \mathrm{~nm}$, respectively. Z-projection images were created using ImageJ software.

\section{Scanning electron microscopy}

Thalli fixed in FAA were dehydrated using a graded ethanol series and isoamyl acetate, and dried using a JCPD-5 critical point dryer (JEOL, Tokyo, Japan). The samples were mounted using carbon tape and coated with platinum using a JEC-3000FC sputter-coater (JEOL). Images of epidermal cell surfaces were captured using a JSM-6510LV scanning electron microscope (JEOL, Tokyo, Japan). Epidermal cell margins were traced using Photoshop software (Adobe) and analyzed using ImageJ software. For observation of antheridiophers, samples were frozen in liquid nitrogen and captured using a VHX-D500 microscope (KEYENCE, Osaka, Japan).

\section{Results}

\section{Liverwort M. polymorpha has a single DYRKP ortholog}

The plant-specific subgroup in the DYRK family, known as the DYRKP subgroup, widely exists from algae to seed plants (Kajikawa et al. 2015; Schulz-Raffelt et al. 2016). Based on a BLAST search and phylogenetic analysis, we identified a single member in the DYRKP subgroup, MpDYRKP (Mp3g19940), in the genome of the basal land plant M. polymorpha (MarpolBase genome database [v5.1: http://marchantia.info]; Fig. 1; Montgomery et al. 2020). MpDYRKP has a highly conserved S/T protein kinase domain in the C-terminal region, which is homologous to other DYRKP orthologs (Fig. 2a). MpDYRKP possesses motifs and amino acids that are important for kinase activity, such as a catalytic loop and a lysine residue, which is a phosphate-anchor to support ATP interactions (Fig. 2b; Aranda et al. 2011). However, although the YxY sequence motif is conserved in the activation loop of the DYRK, DYRK2, and Yak1 subgroups, in the DYRKP subgroup it is conserved as $\mathrm{C} / \mathrm{SxY}$ (Fig. 2b). Transcriptomic analysis of the $M$. polymorpha genome estimated five alternative splicing variants as MpDYRKP gene models (Fig. 3a; Bowman et al. 2017; Montgomery et al. 2020). All alternative splicing variants of MpDYRKP differed within the N-terminal region (Fig. 3a). The N-terminal region of MpDYRKP has no known domains or motifs, as judged by Pfam (http:// pfam.xfam.org). In contrast, the identification of de novo sequence motifs by MEME suites (https://meme-suite.org/ meme/index.html) revealed that there are highly conserved $\mathrm{N}$-terminal region sequence motifs among the DYRKP subgroup (Fig. 2a).

\section{Morphology of the genome-edited Mpdyrkp mutants}

To investigate the roles of MpDYRKP in M. polymor$p h a, \mathrm{Mpdyrkp}$ genome-edited lines were created using the CRISPR/Cas9 system (Sugano et al. 2018). We designed two target sites to obtain independent alleles for deficient mutants, and consequently isolated two genome-edited lines, Mpdyrkp- $1^{g e}$ and Mpdyrkp-2 $2^{g e}$ (Fig. 3b). Mpdyrkp-1 ${ }^{g e}$, which is constructed using gRNA1, has a 41-bp insertion, while Mpdyrkp-2 $2^{g e}$, which is constructed using gRNA2, has a 5-bp substitution and an 11-bp deletion (Fig. 3b).

In 12-day-old plants after gemma germination, both genome-edited lines Mpdyrkp-1 ${ }^{g e}$ and Mpdyrkp-2 ${ }^{g e}$ exhibited growth defects with abnormal thallus morphologies when compared with the wild-type line Tak-1 (Fig. 4). The thalli of both Mpdyrkp mutants were shrunken and less flattened, and their margins were waved (Fig. 4). To confirm the contributions of MpDYRKP to the morphological features of the Mpdyrkp mutants, we constructed a complementation line, pro $\mathrm{Mp} D Y R K P: \mathrm{Mp} D Y R K P-1 / \mathrm{Mp} d y r k p-$ $1^{g e}$. The thallus morphology of this complementation line was restored to that of the wild-type, demonstrating that the abnormal morphologies of the Mpdyrkp gene-edited lines were caused by the loss-of-function of MpDYRKP (Fig. 4). This complementation line uses a $5 \mathrm{kbp}$ region upstream of the start codon as an estimated promoter region and its coding sequence. This promoter region was sufficient to express MpDYRKP as a morphological regulator (Fig. 4). To examine in detail the differences between the wild-type and mutant lines, we traced the development of gemmalings for 12 days (Fig. 5). After day 3, both Mpdyrkp mutants showed vertical growth in their thallus margin when compared with the wild-type and complementation lines (Fig. 5), indicating that the morphological defects in Mpdyrkp are expressed in the early developmental stages of the thallus. M. polymorpha propagates asexually via gemmae formation (Kato et al. 2020; Shimamura 2016). Gemmae are formed at the bottom of the gemma cup, which is a cup-like organ that develops on the middle rib of the thallus. The shape of the gemma cup in Mpdyrkp- $1^{g e}$ was shallow and distorted when compared to the wild-type (Fig. 4). In addition to these morphologies during the vegetative growth phase, morphological differences in the reproductive organs were observed. The male reproductive organs, antheridiophores, of Mpdyrkp-1 $1^{\text {ge }}$ and Mpdyrkp-2 ${ }^{g e}$ showed abnormal morphology with less flattening and waved margins, similar to its thallus morphology (Fig. 6). In addition, the stalks of antheridiophore 


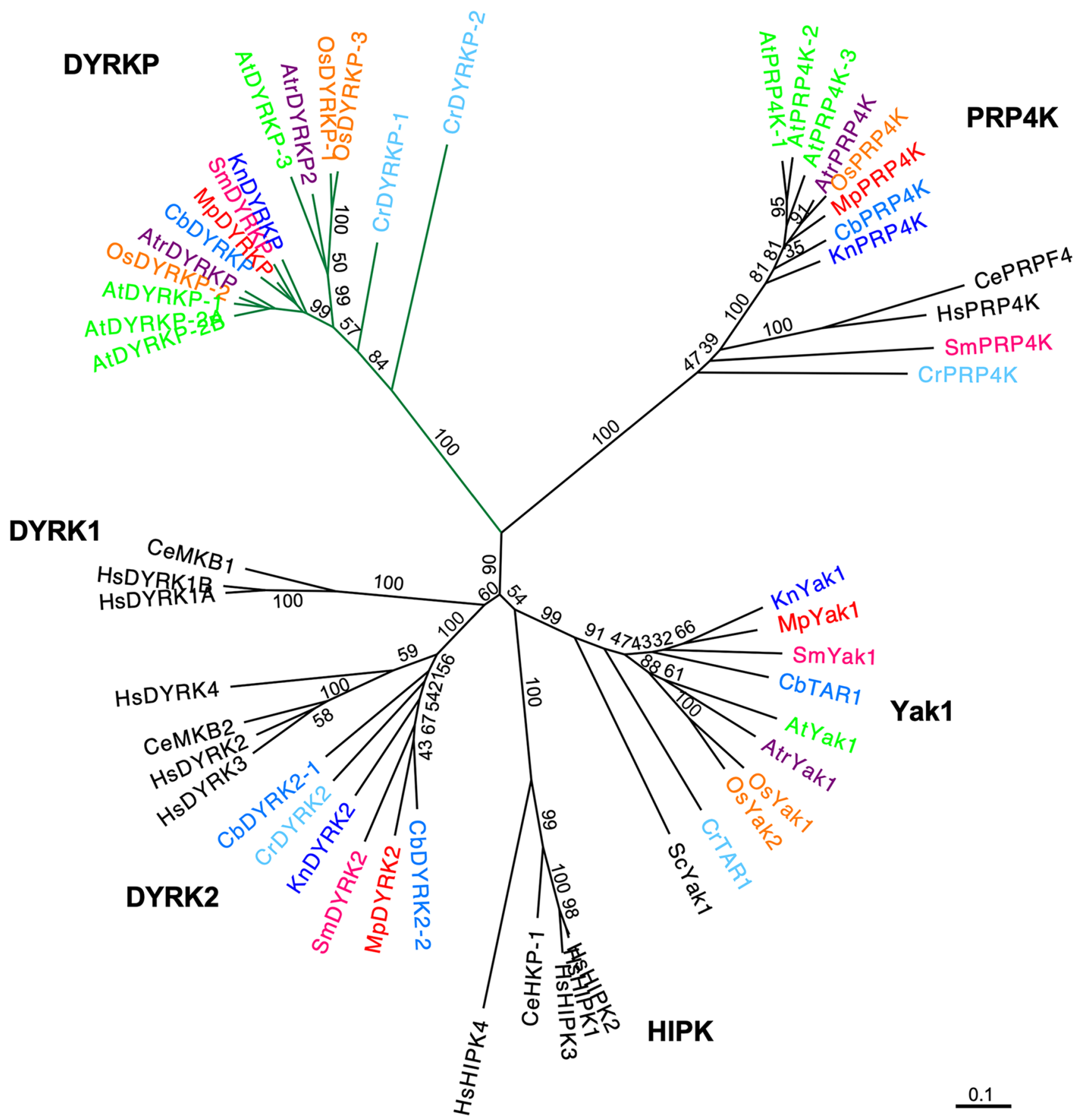

Fig. 1 Phylogenic tree of dual-specificity tyrosine phosphorylationregulated kinases (DYRKs). This phylogenic tree was constructed by comparing the amino acid sequences of the kinase domains from different plant species (Arabidopsis thaliana [At], Oryza sativa [Os], Amborella trichopoda [Atr], Selaginella moellendorffii [Sm], Marchantia polymorpha $[\mathrm{Mp}]$, Chara braunii $[\mathrm{Cb}]$, Klebsormidium nitens $[\mathrm{Kn}]$, and Chlamydomonas reinhardtii $[\mathrm{Cr}]$ ), animals (Caenorhabdi-

of Mpdyrkp- $1^{g e}$ and Mpdyrkp-2 ${ }^{g e}$ were thicker than Tak-1

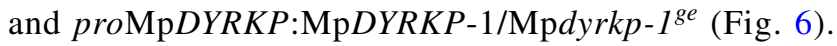
These results suggest that MpDYRKP plays an important role in tissue morphogenesis in both the vegetative and tis elegans [Ce] and Homo sapiens [Hs]), and yeast (Saccharomyces cerevisiae [Sc]). Plant DYRKs are indicated with different colors for each plant species. The plant specific DYRKP subgroup node is green. The tree was generated using MEGA $X$ with the maximum likelihood algorithm. Bootstrap values (1,000 replicates) are indicated at branch nodes, and the scale bar indicates the number of amino acid substitutions per site

reproductive phases. On the other hand, antheridia showed normal development in Mpdyrkp-1 $1^{g e}$ and Mpdyrkp-2 $2^{g e}$ (Fig. 6i-1). 
a

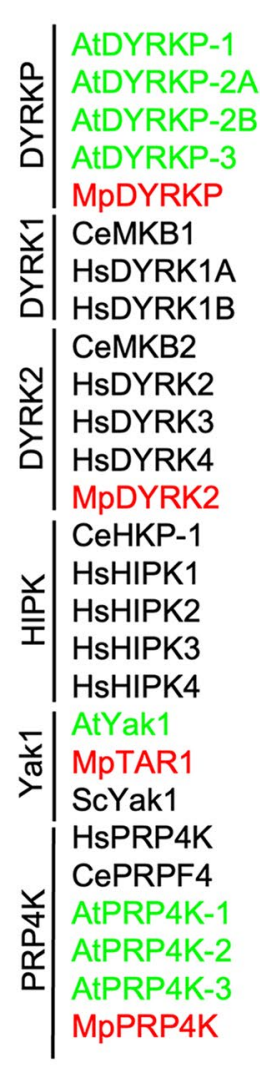

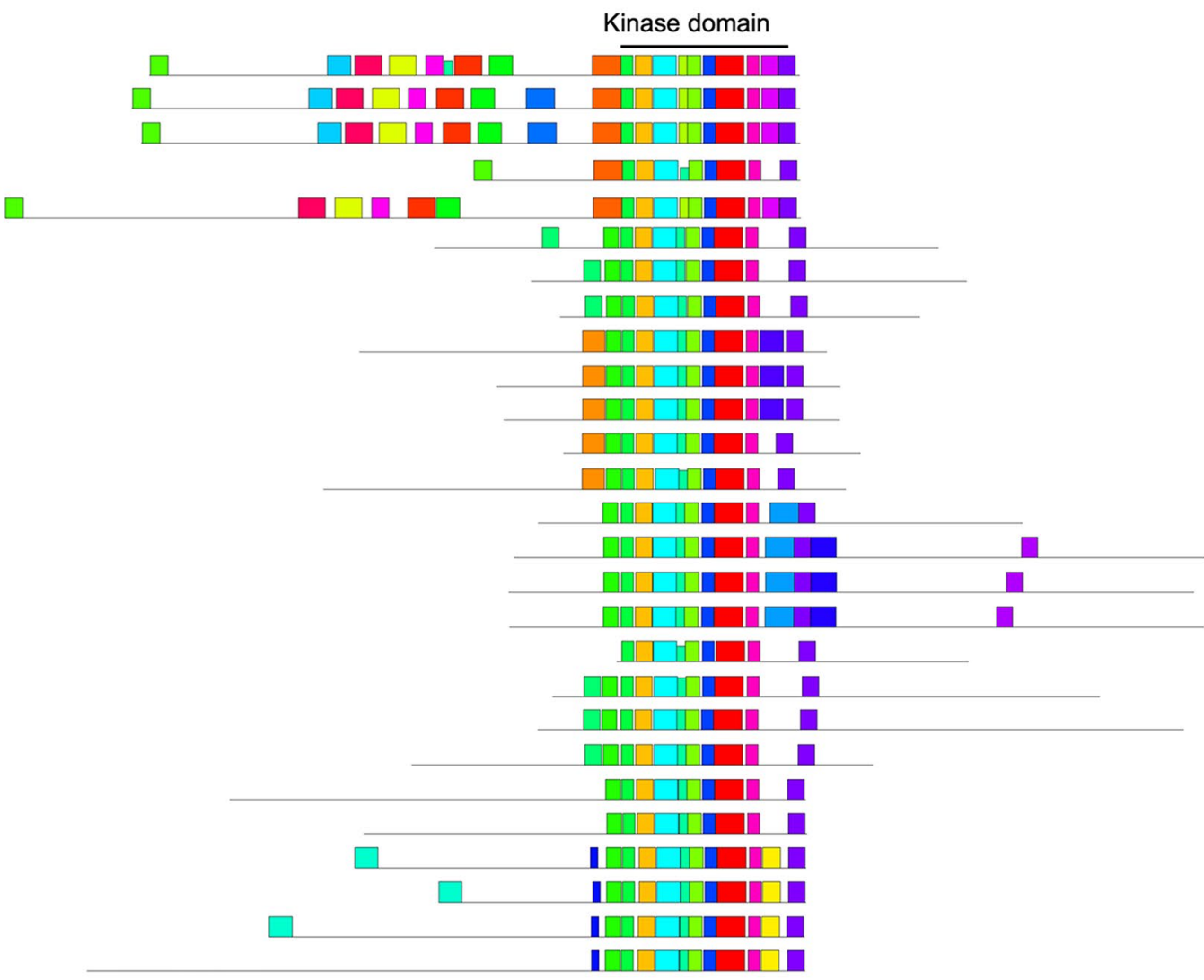

ATP

b 1,120 1,140 1,160 anchor ${ }_{1,180}$

Phosphate anchor

ATDYRKP-1 AtDYRKP-2A - ATDYRKP-2B ATDYRKP-3 - - - - - MPDYRKP - -

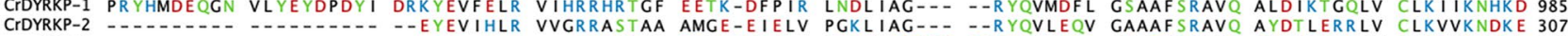
AtYak1 MPTAR1 - MPDYRK2 -

1,220

1,240

1,260

1,280

Catalytic loop

AtDYRKP-1 FFDQSLDEIK LLKYVNQ--H DPADKYHLLR LYDYFYFREH LLIVCELLKA NLYEFQKFNR ESGGEVYFTM PRLQS ITIQC LEALNFLHGL GLIHCDLKPE 961 AtDYRKP-2A FFDQSLDEIK LLKYVNK--H DPADKYHLLR LYDYFYYREH LLIVCELLKA NLYEFHKFNR ESGGEVYFTM PRLQSITIQC LESLQFLHGL GLIHCDLKPE 992 AtDYRKP-2B FFDQSLDEIK LLKYVNK--H DPADKYHLLR LYDYFYYREH LLIVCELLKA NLYEFHKFNR ESGGEVYFTM PRLQS ITIQC LESLQFLHGL GLIHCDLKPE 975

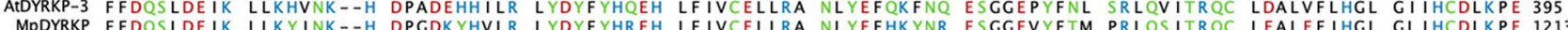

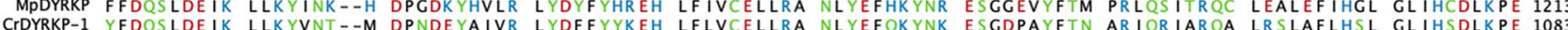

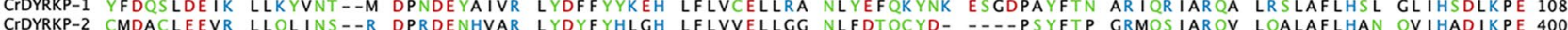

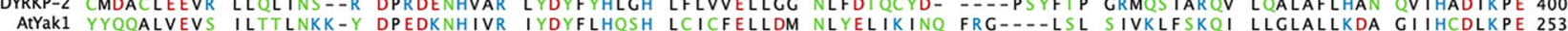

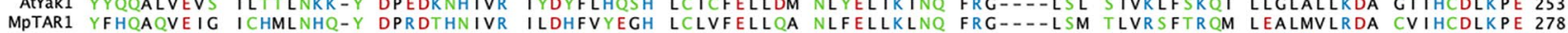
MPDYRK2 FHOQALVEVK ILENLROKAO DEPSNFNIVT IYESFYFRGH LCIAFALHDI SLYELIKRNN FOG---ISL IVIKSFASOL LATLRFLRKL HVIHCDLKPE 656 Cation binding Activation

${ }_{100 p_{1320}} \quad$ loop

1.340

1,360

1.380

1,400

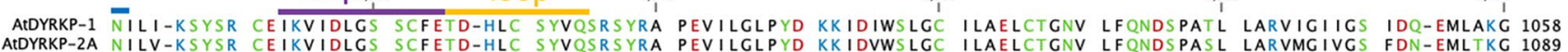
ATDYRP-2B NILV-KSYSR CEIKVIDLGS SCFETD-HLC SYVOSRSYRA PEVILCLPYD KKIDVWSLGC ILAELCTGNV LFONDSPASL LARVMGIVGS FDN-EMLTKG 1089

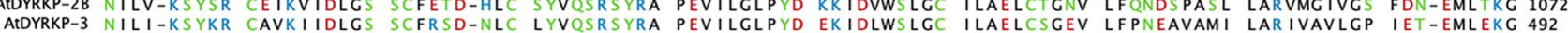
MPDYRKP NILV-KSYSR CEVKVIDLGS SCFOTD-HLC SYVOSRSYRA PEVILGLPYN OKIDMWSLGC ILAELCSGNV LFONDSLATL LARVVGILGP IDP-EMLSKG 1310 CrDYRKP-1 NILI-KSYSR CEVKVIDLGS SCFITD-QLS SYVQSRSYRA PEVILGLPYD YKVDVWSLGC ILAELSSSFV LFONDSLSTL LARLEGILGP VPE-WMLHKG 1180 CrDYRKP-2 NILV-KNYTK CSVKVIDLGC SIYNSEAHLS HYVQSRSYRA PEVMLGLPYD GRIDIWSLGC VMAELATGQV LFPNVSEAAM MARLVGMLGE LPP-YMLQEG 498 AtYak1 NILLCASVKP TEIKIIDFGS ACMEDK-TVY SYIQSRYYRS PEVLLGYQYT TAIDMWSFGC IVAELFLGLP LFPGGSEFDI LRRMIEILGK QPPDYVLKEA 352

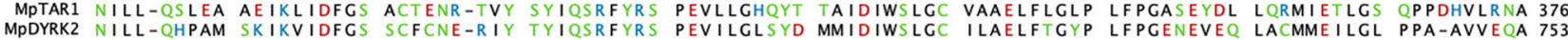

Fig. 2 DYRK amino acid sequence comparison amongst different species. a Sequence motif among DYRKs. Comparison analysis of DYRKs in A. thaliana (At), M. polymorpha (Mp), C. elegans (Ce), and $H$. sapiens $(\mathrm{Hs})$ was preformed using MEME suites. b Multiple sequence alignments were constructed using MUSCLE. Align- ments for a part of the kinase domain of DYRKPs (AtDYRKPs, MpDYRKP, CrDYRKPs), YAK1s (AtYak1 and MpTAR1), and DYRK2 (MpDYRK2) are indicated. Residues were colored according to polarity. Important sequence features for kinase activity are indicated 
a

MpDYRKP

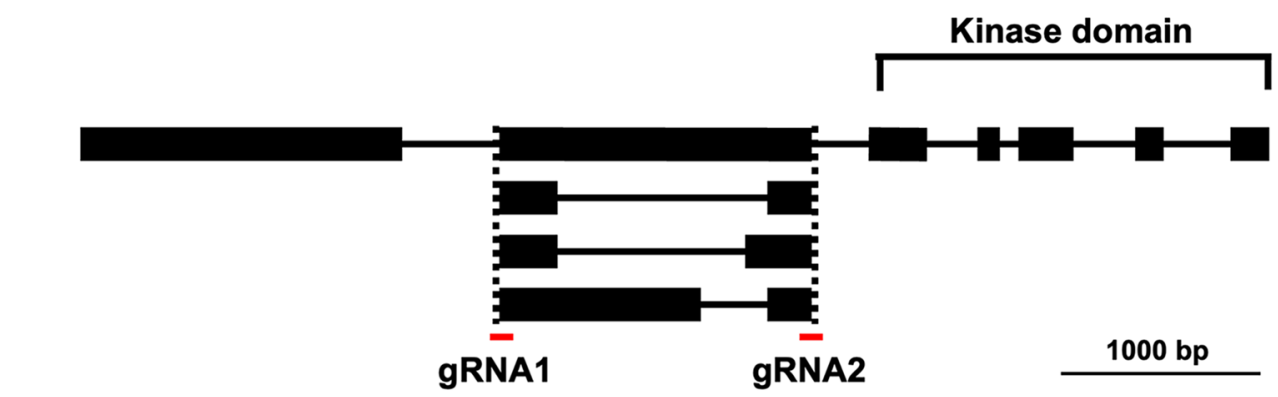

b

Mp3g19940.1

Mp3g19940.2, 4

Mp3g19940.3

Mp3g19940.5

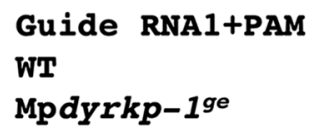

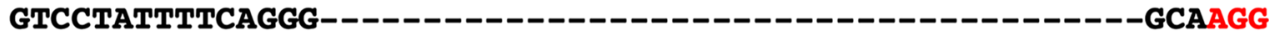

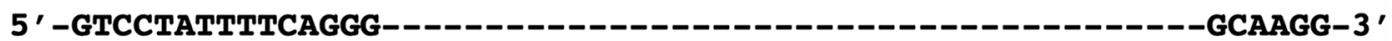

GTCCTATTTTCAGG GECTTATGGATIGCAAGGCTACCTCCACTAAGCCTCCT GCAAGG

intron $1-><-e x o n 2$

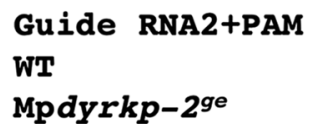

ATCGTACACAGGAAAAACAGG

5 ' -ATCGTACACAGGAAAAACAGG-3'

ATCGT TCTGT----------

exon3-><-1ntron3

Fig. 3 Construction of Mpdyrkp genome-edited lines. a Genomic structure of the encoding region for the MpDYRKP gene. Red bars indicate the locations of the target sites for the gRNAs. b Mutations detected by sequencing analysis. The guide RNA including the PAM

\section{Subcellular localization pattern of MpDYRKP}

To analyze the gene expression pattern of $\mathrm{Mp} D Y R K P$, we re-analyzed the published RNA-seq data sets from various tissues containing thallus, reproductive tissues (antheridiophore, antheridium, and archegoniophore), and developing spores (Bowman et al. 2017; Higo et al. 2016). $\mathrm{MpDYRKP}$ was broadly expressed in all the analyzed tissues (Fig. S1). For detailed expression pattern and protein localization analysis, we constructed a reporter line, proMpDYRKP:MpDYRKP-Citrine-1/Mpdyrkp-1 ${ }^{\text {ge }}$, which used a $5 \mathrm{kbp}$ region upstream from the start codon of $\mathrm{MpDYRKP}$ as a promoter, a process similar to that used for the complementation line. Our observations of this reporter line revealed that MpDYRKP-Citrine was strongly detected around the apical notch (Fig. 7). Moreover, at the subcellular level, MpDYRKP-Citrine was observed as a diffuse punctate structure in the cytosol (Fig. 7). This subcellular localization pattern is different from that of A. thaliana DYRKP-2 A, which is uniformly located in the cytosol with no punctate structure (Iwabuchi et al. 2019).

\section{Cell proliferation activity of Mpdyrkp mutants}

During thallus development, cell proliferation mainly occurrs in the apical notches (Shimamura 2016). Thus, the apical notch is important for thallus morphogenesis. Although the Mpdyrkp gene-edited lines exhibited abnormal sequence (red) and a part of the MpDYRKP sequence in both the wild-type and Mpdyrkp ${ }^{g e}$ lines are shown. The genome-edited line Mpdyrkp- $1^{g e}$ has a 41-bp insertion, while Mpdyrkp-2 $2^{g e}$ has a 5-bp substitution and a 11-bp deletion (orange)

thallus morphology (Figs. 4 and 5), presense of the apical or subapical cells in the apical notch region were recognized in both Mpdyrkp- $1^{g e}$ and Mpdyrkp-2 $2^{g e}$ as well as Tak-1 and proMpDYRKP:MpDYRKP-1/Mpdyrkp-1 ${ }^{\text {ge }}$ (Fig. 8a-d). Next, we measured the number of S-phase cells visualized by EdU staining in the apical notches of 3-day-old plants to assess the cell proliferation activity. The number of EdU signals in the apical notches of Mpdyrkp- $1^{g e}$ was comparable to that of Tak-1 and proMpDYRKP:MpDYRKP-1/Mpdyrkp$1^{g e}$ (Fig. $8 \mathrm{e}-\mathrm{i}$ ). These results suggest that both apical notch formation and cell proliferation activity are not casual factors for the morphological abonormalities in the Mpdyrkp mutants. On the other hand, Mpdyrkp-2 $2^{g e}$ exhibited smaller number of EdU signals in their apical notches compared with Tak-1 (Fig. 8e-i), suggesting that Mpdyrkp-2 ${ }^{\text {ge }}$ may have additional mutations relate to the cell proliferation activity.

\section{Epidermal cell morphology of Mpdyrkp mutants}

The morphological defects in the Mpdyrkp genome-edited lines, suggests that MpDYRKP acts as a morphological regulator (Figs. 4 and 5). As tissue morphogenesis is an accumulation of' cellular morphogenesis, we observed the genome-edited lines, Mpdyrkp- $1^{g e}$ and Mpdyrkp-2 ${ }^{g e}$, using scanning electron microscopy. In contrast to the smooth epidermal surface of Tak-1, the epidermal surfaces of both Mpdyrkp-1 $1^{g e}$ and Mpdyrkp-2 ${ }^{g e}$ appeared uneven and 


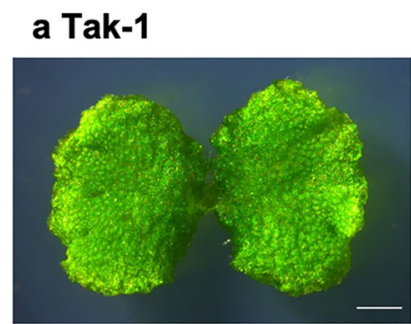

\section{Mpdyrkp-2ge}

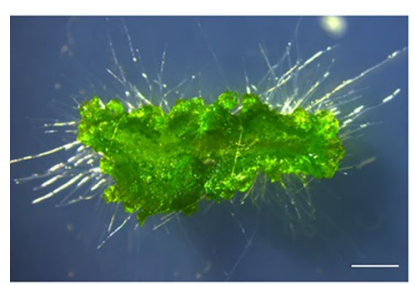

e Tak-1

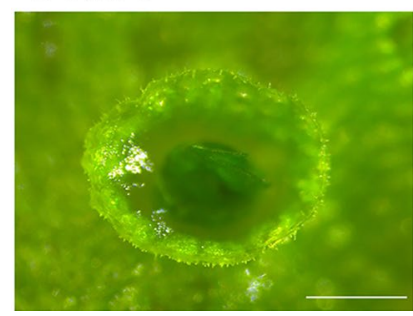

\section{b Mpdyrkp-1ge}

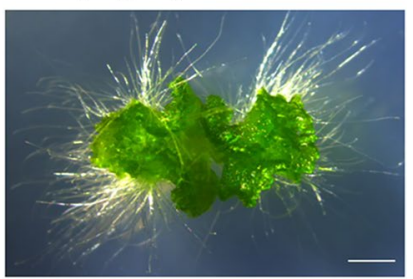

proMpDYRKP:MpDYRKP-1 /dyrkp-19e

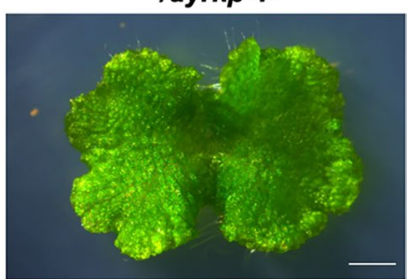

f Mpdyrkp-1ge

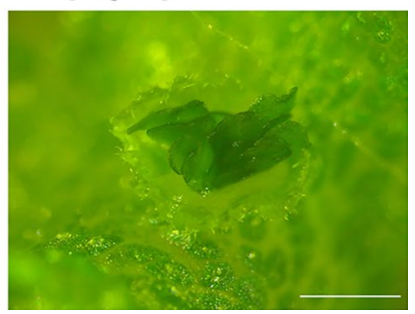

Fig. 4 Morphology of Mpdyrkp mutants. a-d 12-day-old gemmalings of the wild-type line Tak-1 (a); the Mpdyrkp genome-edited lines Mpdyrkp-1 ge $^{g e}$ (b) and Mpdyrkp-2 ${ }^{g e}$ (c); and the complementation line proMpDYRKP:MpDYRKP-1/Mpdyrkp- $1^{g e}$ (d). e-f Gemma cup of Tak-1 (e) and Mpdyrkp-1 ${ }^{\text {ge }}$ (f) cultured for 16 days. Scale bars: $2 \mathrm{~mm}$ (a-d), $1 \mathrm{~mm}(\mathbf{e}, \mathbf{f})$ disordered (Fig. 9a-h). In addition, the shape and size of the air pores in these genome-edited lines were uneven (Fig. 9a-h). Similar trends were observed in the surfaces of antheridial receptacles of both Mpdyrkp- $1^{g e}$ and Mpdyrkp$2^{g e}$ (Fig. 9m-t). Moreover, we found that both Mpdyrkp-1 ${ }^{\text {ge }}$ and Mpdyrkp-2 $2^{g e}$ exhibited a tendency for lower circularity in the epidermal cells of thalli than Tak-1 and the complementation line (Fig. 9i-1). These results suggest that MpDYRKP contributes to thallus morphogenesis, at least in part, via the regulation of epidermal cell shape.

\section{Discussion}

\section{MpDYRKP is involved in the regulation of tissue morphogenesis}

Mpdyrkp genome-edited lines of the model basal land plant M. polymorpha, were shown here to exhibit morphological defects in tissue morphogenesis in both the vegetative and reproductive phases (Figs. 4, 5 and 6). This is the first report regarding the phenotype of a loss-of-function mutant of a DYRKP ortholog in bryophytes. At the cellular level, the epidermal cells were found to have an altered shape with lower circularity in the Mpdyrkp mutants when compared with the wild type (Fig. 9). According to abnormal cell shapes, the epidermal surface of the Mpdyrkp mutants was uneven and disordered (Fig. 9). The disorder in the shape and alignment of the epidermal cells of the Mpdyrkp mutants may account for the morphological disruption of orderly tissues such as the thalli, air pores, and gemma cups (Figs. 4 and 9). These results suggest that MpDYRKP regulates cell

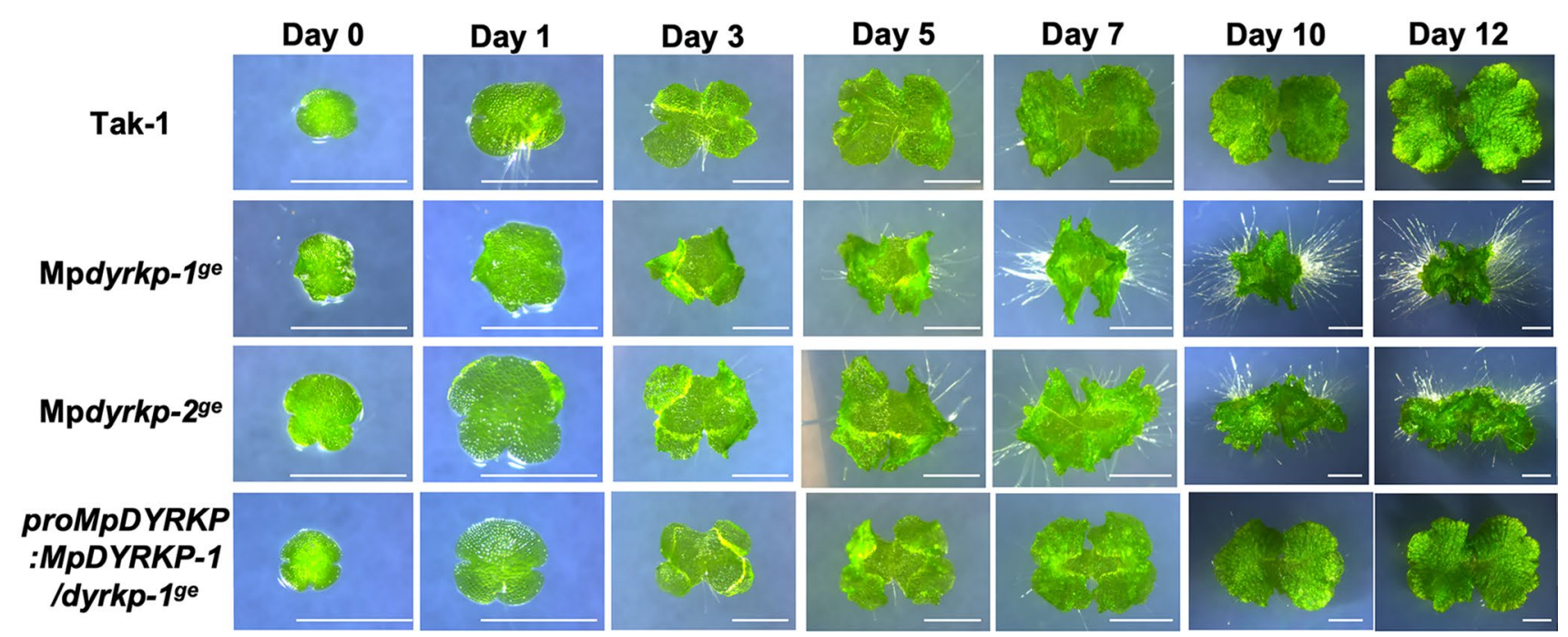

Fig. 5 Time-course observation of Mpdyrkp mutants. The wild-type line Tak-1; the Mpdyrkp genome-edited lines Mpdyrkp-1 $1^{g e}$ and Mpdyrkp-2 $2^{g e}$; and the complementation line;
proMpDYRKP:MpDYRKP-1/Mpdyrkp- $1^{g e}$ were observed at the indicated time points during growth from the gemma. Scale bars: $2 \mathrm{~mm}$ 

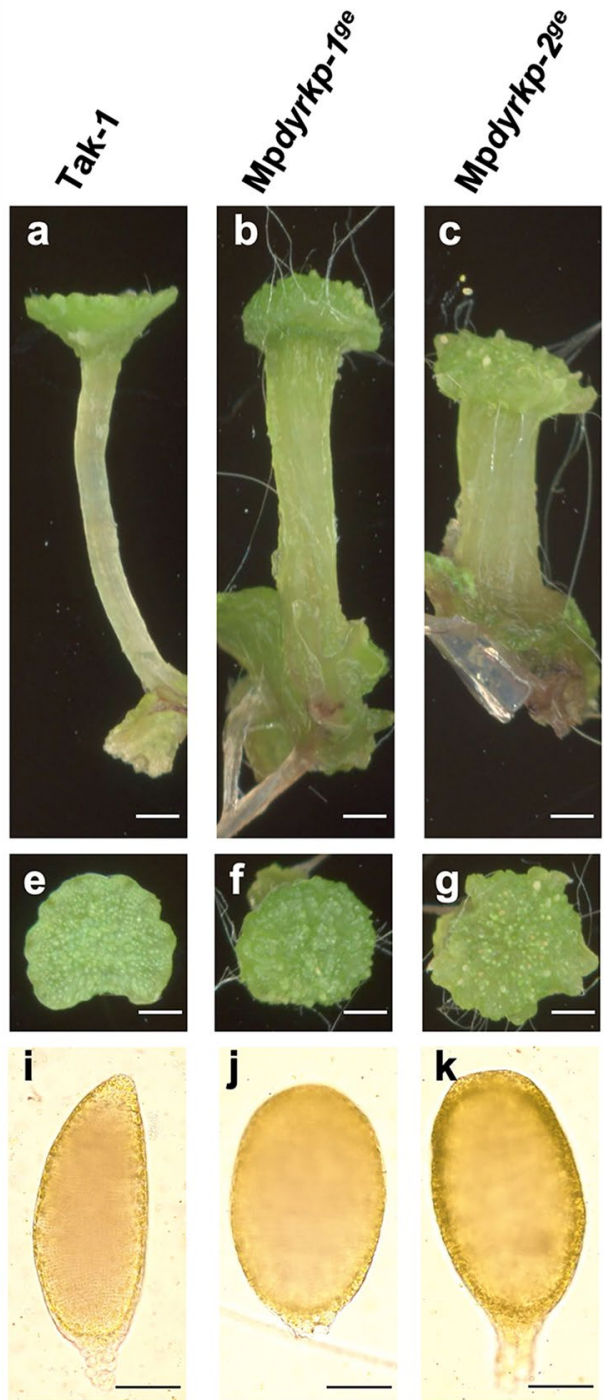
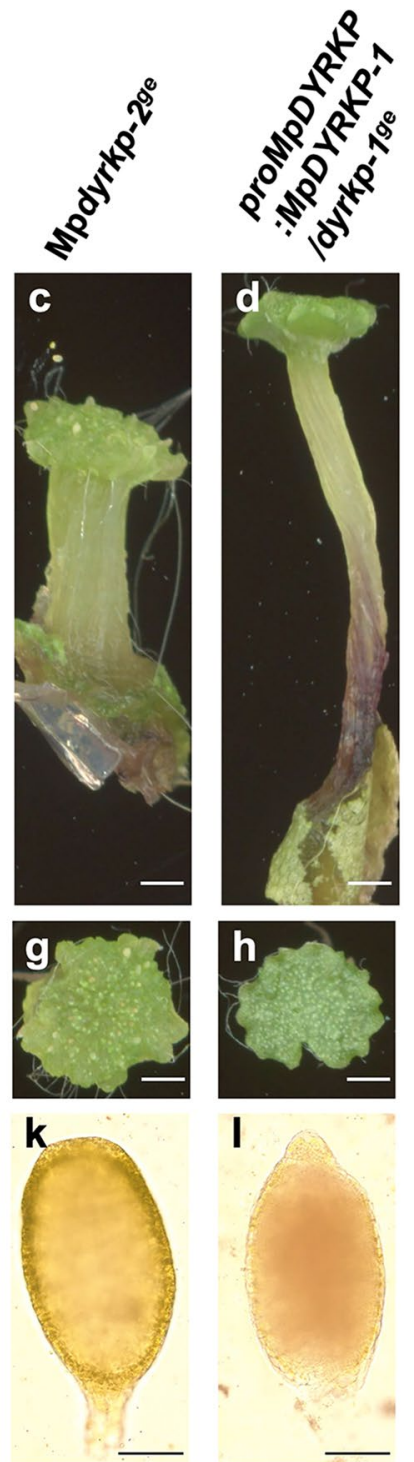

I

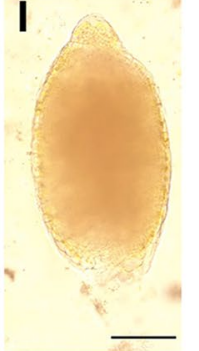

Fig. 6 Morphology of the male reproductive organs of Mpdyrkp mutants. Side (a-d) and top (c-d) views of antheridiophores of Tak-1 (a, e), Mpdyrkp-1 ${ }^{g e}(\mathbf{b}, \mathbf{f}), \operatorname{Mpdyrkp-2^{ge}}$

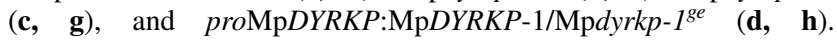
Antheridia of Tak-1 (i), Mpdyrkp-1 ${ }^{\text {ge }}$ (j), Mpdyrkp-2 ${ }^{\text {ge }}$ (k), and proMpDYRKP:MpDYRKP-1/Mpdyrkp- $1^{g e}(\mathbf{l})$. Scale bars: $1 \mathrm{~cm}(\mathbf{a}-\mathbf{h})$, $100 \mu \mathrm{m}(\mathbf{i}-\mathbf{l})$

morphology to enable well-ordered development in M. polymorpha. Generally, cytoskeletons, including microtubules and actin filaments, play important roles in the regulation and determination of plant cell shape (Smith and Oppenheimer 2005). It has been suggested that $A$. thaliana DYRKPs (AtDYRKPs) are involved in nuclear positioning via the regulation of actin filament alignment (Iwabuchi et al. 2019). Moreover, in humans, a member of the DYRK1 subgroup, DYRK1A, induced the destabilization of microtubules via the hyperphosphorylation of tau proteins, which is a causal

pMpDYRKP:MpDYRKP-Citrine-1/dyrkp-1ge

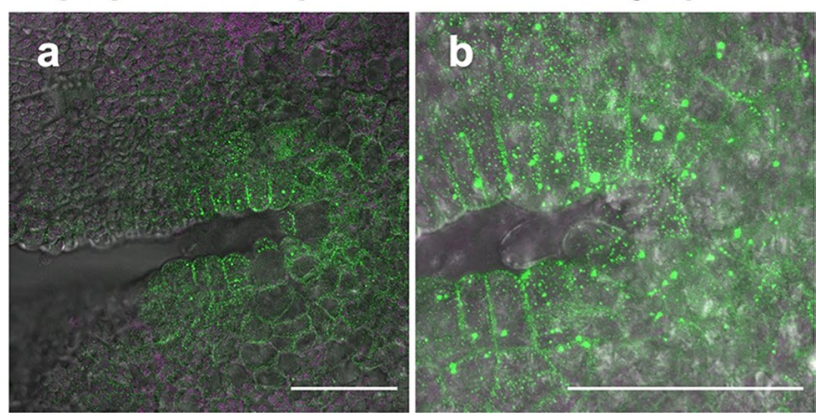

Fig. 7 Subcellular localization of MpDYRKP-Citrine protein. $\mathbf{a}$ and $\mathbf{b}$ Subcellular localization of MpDYRKP-Citrine in 1-day-old gemmal-

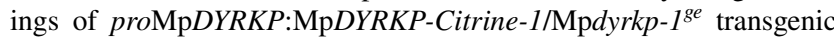
plant was visualized with DIC image Citrine signals are shown in green and autofluorescence in magenta. $\mathbf{b}$ Is a magnified image from around the apical notch in a. Scale bars: $50 \mu \mathrm{m}$

agent of Alzheimer's disease (Ryoo et al. 2007; Woods et al. 2001). Thus, it is important to analyze the molecular functions and identify the interacting proteins of MpDYRKP to understand the underlying mechanisms of tissue morphogenesis in M. polymorpha.

\section{Relationship between DYRKPs and AN}

In A. thaliana, DYRKPs were found to interact with AN proteins in the yeast two-hybrid screens (Bhasin and Hülskamp 2017: ANGSTIFOLIA INTERACTING KINASE [AIK1] corresponds to AtDYRKP-1), and the co-immunoprecipitation assay using transgenic plants expressing AN-GFP (Iwabuchi et al. 2019). Mutants deficient in AN exhibited multiple morphological phenotypes, such as narrow and thicker leaves, twisted fruits and petals, and the premature opening of flowers (Bai et al. 2010, 2013; Rédei 1962; Tsuge et al. 1996; Tsukaya et al. 1994). In M. polymorpha, the knockout mutants of the AN ortholog, Mpan, showed abnormal twisted thalli and suppressed thallus growth, indicating that MpAN is also involved in the regulation of tissue morphogenesis (Furuya et al. 2018). Moreover, in both $A$. thaliana and M. polymorpha, the loss of function of AN results in an abnormal arrangement of the cortical microtubules (Furuya et al. 2018; Kim et al. 2002). The phenotypes between Mpdyrkp and Mpan mutants were partially shared in the context of abnormal morphologies with less flattening of the thalli and antheridial receptacles (Figs. 4, 5 and 6). However, it is not easy to compare the phenotypic similarities between the Mpdyrkp and Mpan mutants due to their morphological complexity. In terms of epidermal cell shapes, Mpdyrkp showed lower circularity than the wild type under normal growth conditions (Fig. 9), whereas Mpan showed higher circularity only under weak blue light conditions (Furuya et al. 2018). These results indicate that the 
a Tak-1

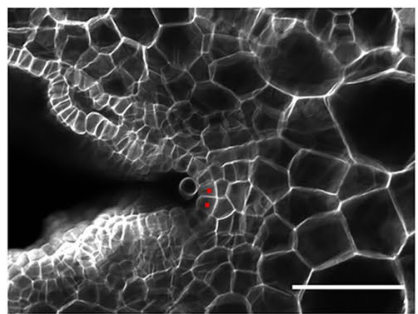

e Tak-1

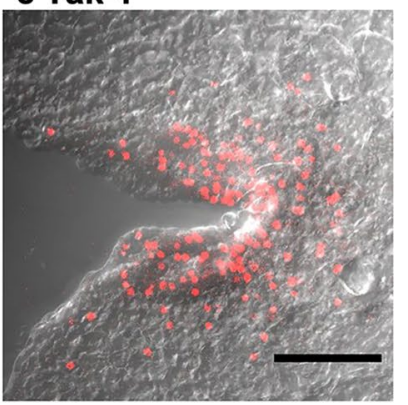

g Mpdyrkp-2ge

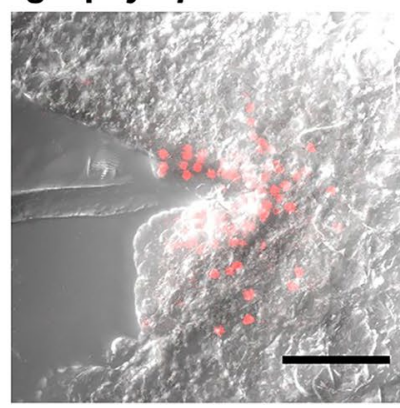

b Mpdyrkp-19e

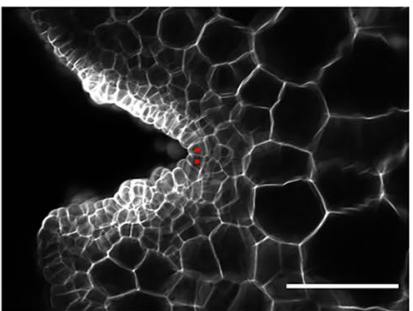

f Mpdyrkp-1ge

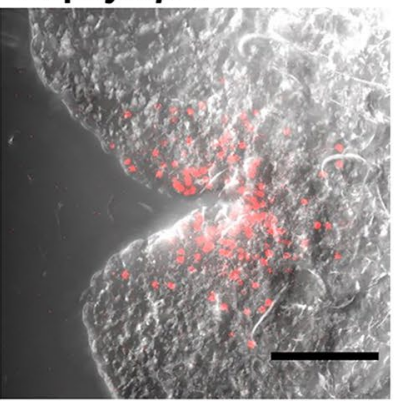

h proMpDYRKP:MpDYRKP-1 /dyrkp-19e

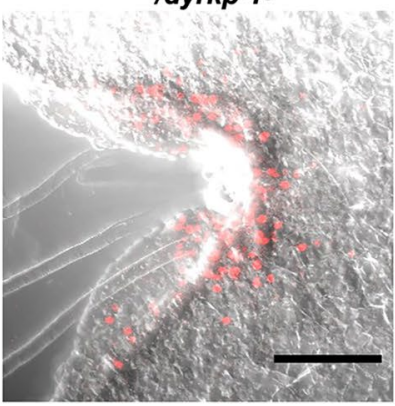

C Mpdyrkp-2ge

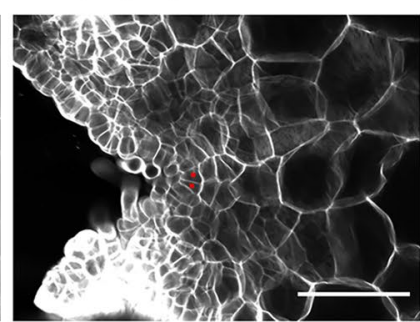

d ProMpDYRKP:MpDYRKP-1

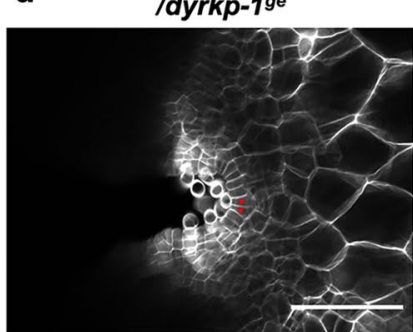

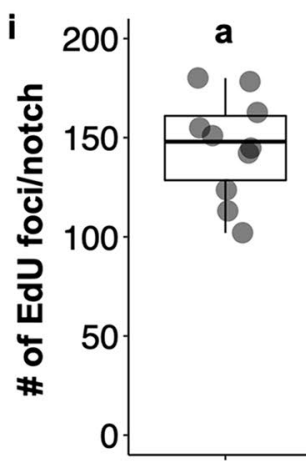

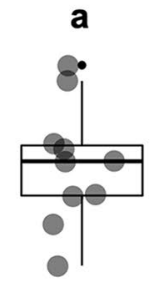

a
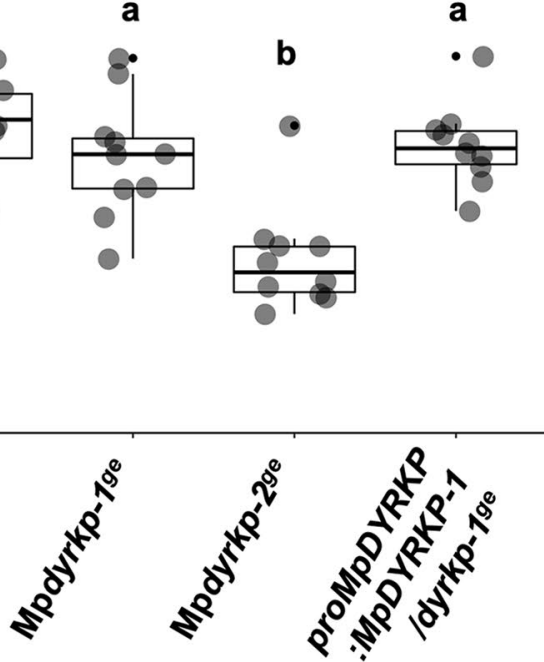

Fig. 8 Cell proliferation activity in Mpdyrkp mutants. a-d Conforcal images of the apical notches in the Tak-1 (a), Mpdyrkp-1 ${ }^{g e}$ (b), Mpdyrkp-2 ${ }^{g e}$ (c), and proMpDYRKP:MpDYRKP-1/Mpdyrkp- $1^{g e}$ (d). Plants cultured for 3 days after gemma germination were treated with ClearSee solution. Cell walls stained by SCRI Renaissance 2200 are shown in white. Red dots indicate apical and/or subapical cells. e-h S-phase nuclei visualized by EdU in the Tak-1 (e), Mpdyrkp-1 $1^{g e}$ (f),

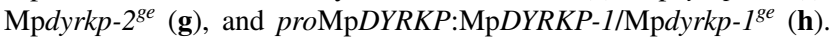
Plants cultured for 3 days after gemma germination were treated with EdU-containing liquid medium for $1 \mathrm{~h}$. Z-series fluorescence images

effects of the Mpdyrkp mutation are more severe and opposing to those of the Mpan mutation. The protein localization of MpDYRKP-Citrine was observed as a diffuse punctate structure in the cytosol (Fig. 7). This localization pattern is different from that of GFP-DYRKP-2 A in A. thaliana, which is uniformly located in the cytosol with no punctate structure (Iwabuchi et al. 2019). Interestingly, the diffuse punctate structures observed in MpDYRKP-Citrine transgenic plants were similar to the AN protein localization pattern observed in both $M$. polymorpha and A. thaliana (Bhasin and Hülskamp 2017; Furuya et al. 2018). Further studies of the molecular and genetic interactions between

DYRKP and AN in M. polymorpha will provide clues to uncover their functional mechanisms in tissue and cell morphogenesis.

\section{Conclusions}

In this study, we revealed that MpDYRKP is involved in the regulation of cell shape to enable the formation of orderly tissue morphologies in M. polymorpha. However, the molecular function, target substrates, and physiological roles of MpDYRKP and other DYRKPs remain unknown. 


\section{Tak-1}
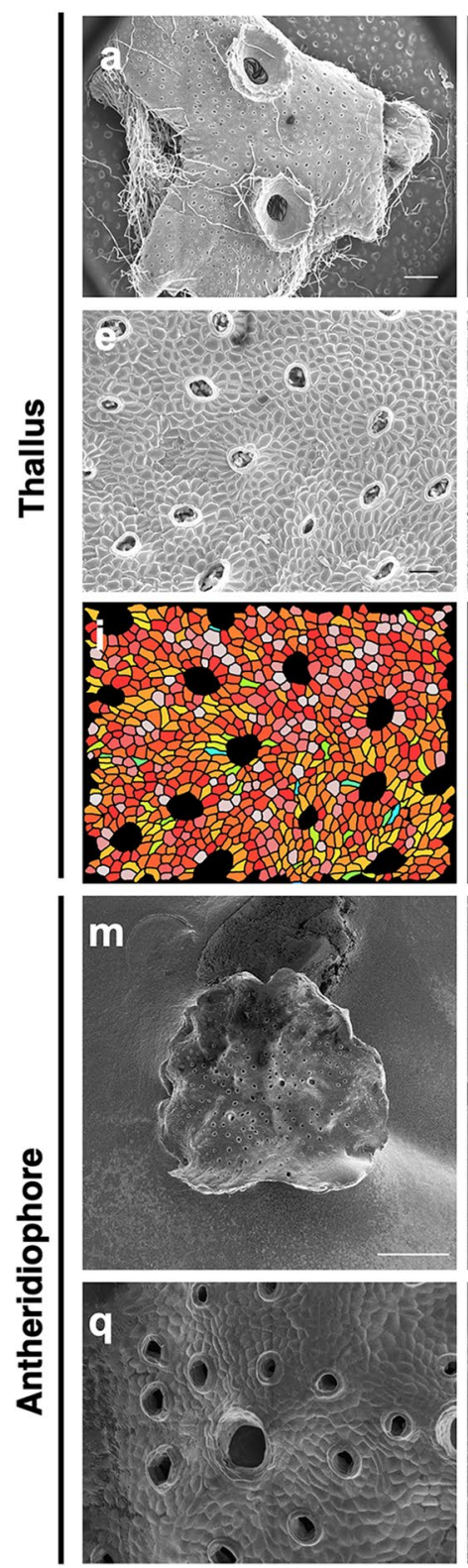

Mpdyrkp-1ge
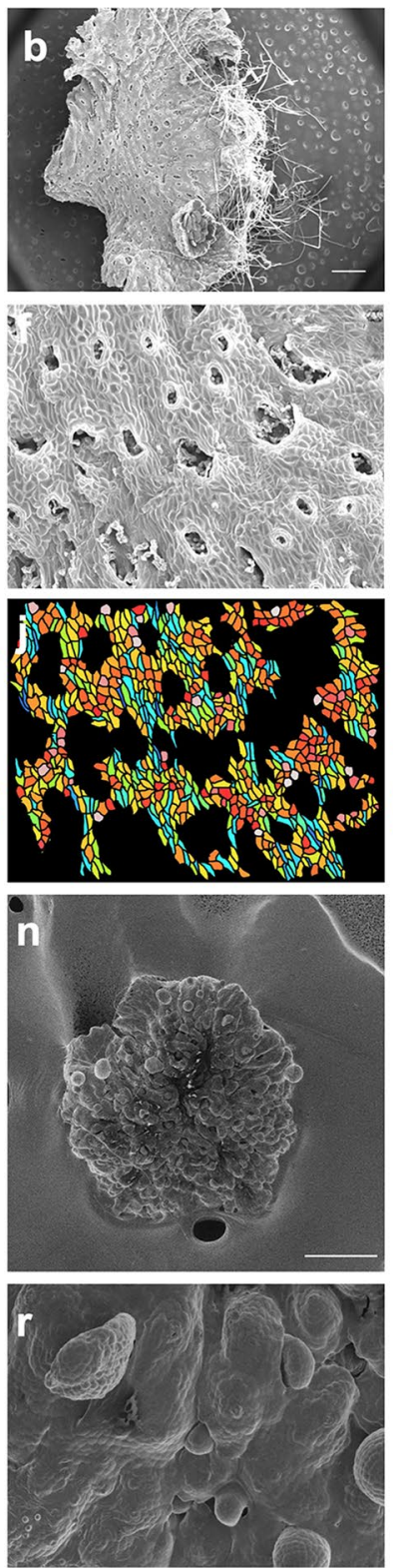

Fig. 9 Epidermal morphology in Mpdyrkp mutants. a-h Scanning electron microscopy images of thalli of Tak-1 (a, e), Mpdyrkp- $1^{\text {ge }}$ (b,

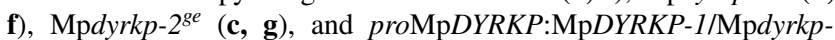
$1^{g e}(\mathbf{d}, \mathbf{h})$. i-l Epidermal-cell circularity shown in scanning electron microscopy images (e-h) were measured using Image J. m-t

Comparison of the DYRKPs revealed that features of the amino acid sequences of MpDYRKP are conserved across a broad range of plant species. Thus, the molecular functions of MpDYRKP, as a morphological regulator in M. polymor$p h a$, may have been conserved during plant evolution.

Supplementary Information The online version contains supplementary material available at https://doi.org/10.1007/s10265-021-01345-w.
Mpdyrkp-2ge
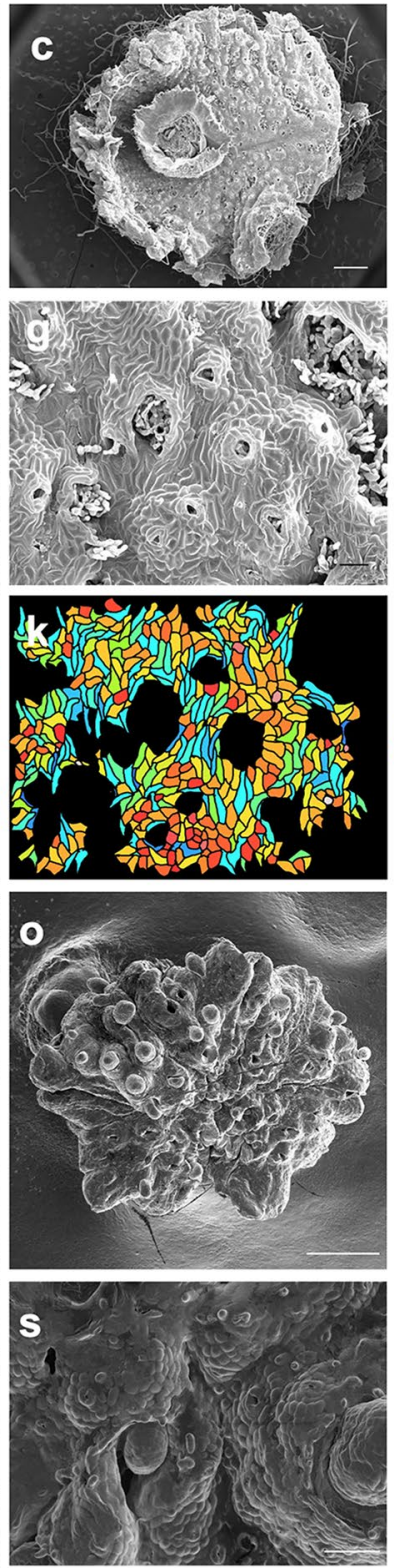

proMpDYRKP:MpDYRKP-1

/dyrkp-1ge
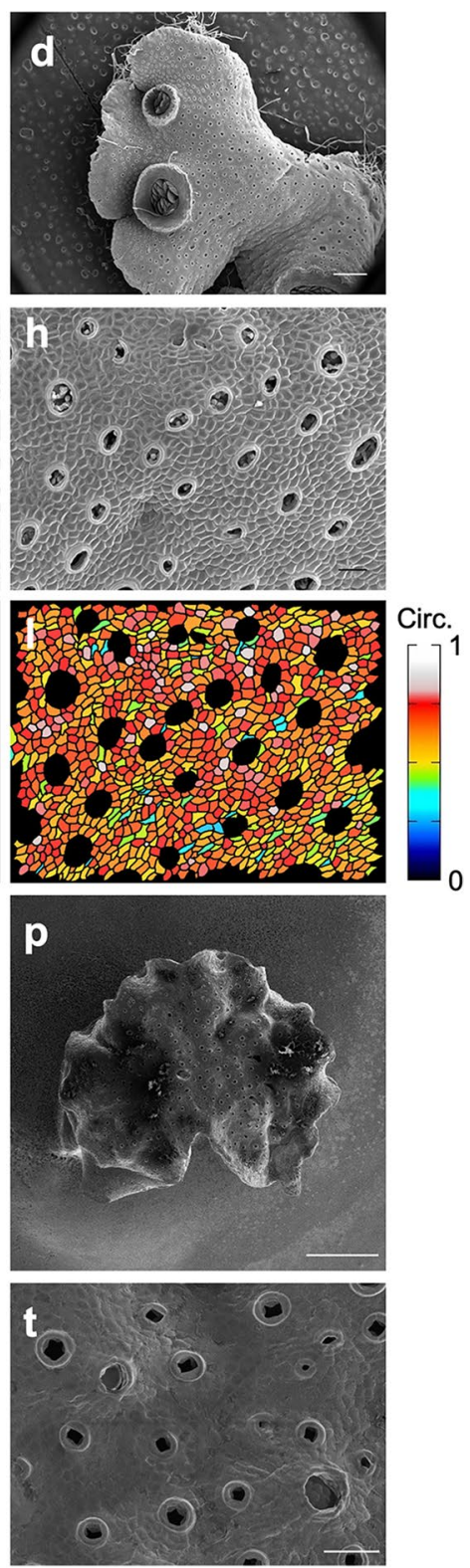

Scanning electron microscopy images of antheridial receptacles of Tak-1 (m and q), Mpdyrkp-1 ${ }^{g e}(\mathbf{n}$ and $\mathbf{r}), \operatorname{Mpdyrkp-2^{ge}}(\mathbf{o}, \mathbf{s})$, and proMpDYRKP:MpDYRKP-1/Mpdyrkp-1 ${ }^{g e}(\mathbf{p}, \mathbf{t})$. Scale bars: $1 \mathrm{~mm}$ $(\mathbf{a}-\mathbf{d}, \mathbf{m}-\mathbf{p}), 100 \mu \mathrm{m}(\mathbf{e}-\mathbf{h}, \mathbf{q}-\mathbf{t})$

Acknowledgements We thank Dr. Yuki Kondo (Kobe University) for his helpful and kind support, Yoriko Matsuda (Kyoto University) for her support with the mutant isolation by genome editing, and Dr. Yuuki Sakai and Dr. Kimitsune Ishizaki (Kobe University) for their technical support for the observation using the scanning electron and confocal microscopy and the EdU staining. This work was funded by the Japan Society for the Promotion of Science KAKENHI EST (19K21189 and $20 \mathrm{~K} 15813$ to T.F., $17 \mathrm{~K} 07753$ to M.K., 16 H04805 to H.F.), and a Grantin-Aid for Scientific Research on Innovation Areas from Ministry of 
Education, Culture, Sports, Science and Technology (25113002 and 19H05672 to H.T.,251113009, 25113001, and 19H05675 to TK).

Open Access This article is licensed under a Creative Commons Attribution 4.0 International License, which permits use, sharing, adaptation, distribution and reproduction in any medium or format, as long as you give appropriate credit to the original author(s) and the source, provide a link to the Creative Commons licence, and indicate if changes were made. The images or other third party material in this article are included in the article's Creative Commons licence, unless indicated otherwise in a credit line to the material. If material is not included in the article's Creative Commons licence and your intended use is not permitted by statutory regulation or exceeds the permitted use, you will need to obtain permission directly from the copyright holder. To view a copy of this licence, visit http://creativecommons.org/licenses/by/4.0/.

\section{References}

Aranda S, Laguna A, de la Luna S (2011) DYRK family of protein kinases: evolutionary relationships, biochemical properties, and functional roles. FASEB J 25:449-462. doi:https://doi.org/10. 1096/fj.10-165837

Bai Y, Falk S, Schnittger A, Jakoby MJ, Hülskamp M (2010) Tissue layer specific regulation of leaf length and width in Arabidopsis as revealed by the cell autonomous action of ANGUSTIFOLIA. Plant J 61:191-199. doi:https://doi.org/10.1111/j.1365-313X. 2009.04050.x

Bai Y, Vaddepalli P, Fulton L, Bhasin H, Hülskamp M, Schneitz K (2013) ANGUSTIFOLIA is a central component of tissue morphogenesis mediated by the atypical receptor-like kinase STRUBBELIG. BMC Plant Biol 13:16. doi:https://doi.org/10.1186/ 1471-2229-13-16

Barrada A, Djendli M, Desnos T, Mercier R, Robaglia C, Montané MH, Menand B (2019) A TOR-YAK1 signaling axis controls cell cycle, meristem activity and plant growth in Arabidopsis. Development 146:dev171298. doi:https://doi.org/10.1242/dev.171298

Becker W (2012) Emerging role of DYRK family protein kinases as regulators of protein stability in cell cycle control. Cell Cycle 11:3389-3394. doi:https://doi.org/10.4161/cc.21404

Bhasin H, Hülskamp M (2017) ANGUSTIFOLIA, a plant homolog of CtBP/BARS localizes to stress granules and regulates their formation. Front Plant Sci 8:1004. doi:https://doi.org/10.3389/ fpls.2017.01004

Bowman JL et al (2017) Insights into land plant evolution garnered from the Marchantia polymorpha genome. Cell 171:287-304.e15. doi:https://doi.org/10.1016/j.cell.2017.09.030

Forzani C, Duarte GT, Van Leene J, Clément G, Huguet S, Paysant-LeRoux C, Mercier R, De Jaeger G, Leprince AS, Meyer C (2019) Mutations of the AtYAK1 kinase suppress TOR deficiency in Arabidopsis. Cell Rep 27:3696-3708.e5. https://doi.org/10.1016/j. celrep.2019.05.074

Furuya T, Hattori K, Kimori Y, Ishida S, Nishihama R, Kohchi T, Tsukaya H (2018) ANGUSTIFOLIA contributes to the regulation of three-dimensional morphogenesis in the liverwort Marchantia polymorpha. Development 145:dev161398. doi:https://doi.org/10. 1242/dev.161398

Gamborg OL, Miller RA, Ojima K (1968) Nutrient requirements of suspension cultures of soybean root cells. Exp Cell Res 50:151158. doi:https://doi.org/10.1016/0014-4827(68)90403-5

Garrett S, Broach J (1989) Loss of Ras activity in Saccharomyces cerevisiae is suppressed by disruptions of a new kinase gene, YAKI, whose product may act downstream of the cAMP-dependent protein kinase. Genes Dev 3:1336-1348. doi:https://doi.org/10. 1101/gad.3.9.1336

Gwack Y, Sharma S, Nardone J, Tanasa B, Iuga A, Srikanth S, Okamura H, Bolton D, Feske S, Hogan PG, Rao A (2006) A genomewide Drosophila RNAi screen identifies DYRK-family kinases as regulators of NFAT. Nature 441:646-650. doi:https://doi.org/ 10.1038/nature04631

Higo A, Niwa M, Yamato KT, Yamada L, Sawada H, Sakamoto T, Kurata T, Shirakawa M, Endo M, Shigenobu S, Yamaguchi K, Ishizaki K, Nishihama R, Kohchi T, Araki T (2016) Transcriptional framework of male gametogenesis in the liverwort Marchantia polymorpha L. Plant Cell Physiol 57:325-338. doi:https:// doi.org/10.1093/pcp/pcw005

Huang WY, Wu YC, Pu HY, Wang Y, Jang GJ, Wu SH (2017) Plant dual-specificity tyrosine phosphorylation-regulated kinase optimizes light-regulated growth and development in Arabidopsis. Plant Cell Environ 40:1735-1747. doi:https://doi.org/10.1111/ pce. 12977

Ishizaki K, Chiyoda S, Yamato KT, Kohchi T (2008) Agrobacterium mediated transformation of the haploid liverwort Marchantia polymorpha $\mathrm{L}$., an emerging model for plant biology. Plant Cell Physiol 49:1084-1091. https://doi.org/10.1093/pcp/pcn085

Ishizaki K, Nishihama R, Ueda M, Inoue K, Ishida S, Nishimura Y, Shikanai T, Kohchi T (2015) Development of gateway binary vector series with four different selection markers for the liverwort Marchantia polymorpha. PLoS One 10:e138876. doi:https://doi.org/10.1371/journal.pone.0138876

Ishizaki K, Nishihama R, Yamato KT, Kohchi T (2016) Molecular genetic tools and techniques for Marchantia polymorpha research. Plant Cell Physiol 57:262-270. doi:https://doi.org/10. 1093/pcp/pcv097

Iwabuchi K, Ohnishi H, Tamura K, Fukao Y, Furuya T, Hattori K, Tsukaya H, Hara-Nishimura I (2019) ANGUSTIFOLIA regulates actin filament alignment for nuclear positioning in leaves. Plant Physiol 179:233-247. doi:https://doi.org/10.1104/pp.18. 01150

Kajikawa M, Sawaragi Y, Shinkawa H, Yamano T, Ando A, Kato M, Hirono M, Sato N, Fukuzawa H (2015) Algal dual-specificity tyrosine phosphorylation-regulated kinase, triacylglycerol accumulation regulator1, regulates accumulation of triacylglycerol in nitrogen or sulfur deficiency. Plant Physiol 168:752-764. doi:https://doi.org/10.1104/pp.15.00319

Kato H, Yasui Y, Ishizaki K (2020) Gemma cup and gemma development in Marchantia polymorpha. New Phytol 228:459-465. doi:https://doi.org/10.1111/nph.16655

Kim GT, Shoda K, Tsuge T, Cho KH, Uchimiya H, Yokoyama R, Nishitani K, Tsukaya H (2002) The ANGUSTIFOLIA gene of Arabidopsis, a plant $C t B P$ gene, regulates leaf-cell expansion, the arrangement of cortical microtubules in leaf cells and expression of a gene involved in cell-wall formation. EMBO J 21:1267-1279. doi:https://doi.org/10.1093/emboj/21.6.1267

Kim D, Ntui VO, Xiong L (2016) Arabidopsis YAK1 regulates abscisic acid response and drought resistance. FEBS Lett 590:2201-2209. doi:https://doi.org/10.1002/1873-3468.12234

Kohchi T, Yamato KT, Ishizaki K, Yamaoka S, Nishihama R (2021) Development and molecular genetics of Marchantia polymorpha. Annu Rev Plant Biol. doi:https://doi.org/10.1146/annurev-arpla nt-082520-094256

Kubota A, Ishizaki K, Hosaka M, Kohchi T (2013) Efficient Agrobacterium-mediated transformation of the liverwort Marchantia polymorpha using regenerating thalli. Biosci Biotechnol Biochem 77:167-172. doi:https://doi.org/10.1271/bbb.120700

Kumar S, Stecher G, Li M, Knyaz C, Tamura K (2018) MEGA X: molecular evolutionary genetics analysis across computing platforms. Mol Biol Evol 35:1547-1549. https://doi.org/10.1093/ molbev/msy096 
Kurabayashi N, Sanada K (2013) Increased dosage of DYRK1A and DSCR1 delays neuronal differentiation in neocortical progenitor cells. Genes Dev 27:2708-2721. doi:https://doi.org/10.1101/gad. 226381.113

Kurihara D, Mizuta Y, Sato Y, Higashiyama T (2015) ClearSee: a rapid optical clearing reagent for whole-plant fluorescence imaging. Development 142:4168-4179. doi:https://doi.org/10.1242/ dev. 127613

Lochhead PA, Sibbet G, Morrice N, Cleghon V (2005) Activation-loop autophosphorylation is mediated by a novel transitional intermediate form of DYRKs. Cell 121:925-936. doi:https://doi.org/10. 1016/j.cell.2005.03.034

Manning G, Whyte DB, Martinez R, Hunter T, Sudarsanam S (2002) The protein kinase complement of the human genome. Science 298:1912-1934. doi:https://doi.org/10.1126/science.1075762

Montgomery SA et al (2020) Chromatin organization in early land plants reveals an ancestral association between $\mathrm{H} 3 \mathrm{~K} 27 \mathrm{me} 3$, transposons, and constitutive heterochromatin. Curr Biol 30:573-588. e7. doi:https://doi.org/10.1016/j.cub.2019.12.015

Motohashi K (2015) A simple and efficient seamless DNA cloning method using SLiCE from emopenEscherichia coliemclose laboratory strains and its application to SliP site-directed mutagenesis. BMC Biotechnol 15:47. https://doi.org/10.1186/ s12896-015-0162-8

Naramoto S, Jones VAS, Trozzi N, Sato M, Toyooka K, Shimamura M, Ishida S, Nishitani K, Ishizaki K, Nishihama R, Kohchi T, Dolan L, Kyozuka J (2019) A conserved regulatory mechanism mediates the convergent evolution of plant shoot lateral organs. PLoS Biol 17:e3000560. https://doi.org/10.1371/journal.pbio.3000560

Nihira NT, Yoshida K (2015) Engagement of DYRK2 in proper control for cell division. Cell Cycle 14:802-807. doi:https://doi.org/10. 1080/15384101.2015.1007751

Nishihama R, Ishizaki K, Hosaka M, Matsuda Y, Kubota A, Kohchi $\mathrm{T}$ (2015) Phytochrome-mediated regulation of cell division and growth during regeneration and sporeling development in the liverwort Marchantia polymorpha. J Plant Res 128:407-421. doi:https://doi.org/10.1007/s10265-015-0724-9

Okada S, Fujisawa M, Sone T, Nakayama S, Nishiyama R, Takenaka M, Yamaoka S, Sakaida M, Kono K, Takahama M, Yamato KT, Fukuzawa H, Brennicke A, Ohyama K (2000) Construction of male and female PAC genomic libraries suitable for identification of Y-chromosome-specific clones from the liverwort, Marchantia polymorpha. Plant J 24:421-428. doi:https://doi.org/10.1046/j. 1365-313x.2000.00882.x

Rédei GP (1962) Supervital mutants of Arabidopsis. Genetics 47:443-460

Ryoo SR, Jeong HK, Radnaabazar C, Yoo JJ, Cho HJ, Lee HW, Kim IS, Cheon YH, Ahn YS, Chung SH, Song WJ (2007) DYRK1Amediated hyperphosphorylation of Tau. A functional link between Down syndrome and Alzheimer disease. J Biol Chem 282:34850 34857. doi:https://doi.org/10.1074/jbc.M707358200

Schulz-Raffelt M, Chochois V, Auroy P, Cuiné S, Billon E, Dauvillée D, Li-Beisson Y, Peltier G (2016) Hyper-accumulation of starch and oil in a Chlamydomonas mutant affected in a plant-specific DYRK kinase. Biotechnol Biofuels 9:55. https://doi.org/10.1186/ s13068-016-0469-2

Shimamura M (2016) Marchantia polymorpha: taxonomy, phylogeny and morphology of a model system. Plant Cell Physiol 57:230 256. doi:https://doi.org/10.1093/pcp/pcv192

Smith LG, Oppenheimer DG (2005) Spatial control of cell expansion by the plant cytoskeleton. Annu Rev Cell Dev Biol 21:271-295. doi:https://doi.org/10.1146/annurev.cellbio.21.122303.114901

Soppa U, Becker W (2015) DYRK protein kinases. Curr Biol 25:R488R489. doi:https://doi.org/10.1016/j.cub.2015.02.067

Sugano SS, Nishihama R, Shirakawa M, Takagi J, Matsuda Y, Ishida S, Shimada T, Hara-Nishimura I, Osakabe K, Kohchi T (2018) Efficient CRISPR/Cas9-based genome editing and its application to conditional genetic analysis in Marchantia polymorpha. PLoS One 13:e0205117. doi:https://doi.org/10.1371/journal.pone.02051 17

Taira N, Nihira K, Yamaguchi T, Miki Y, Yoshida K (2007) DYRK2 is targeted to the nucleus and controls p53 via Ser46 phosphorylation in the apoptotic response to DNA damage. Mol Cell 25:725738. https://doi.org/10.1016/j.molcel.2007.02.007

Taira N, Mimoto R, Kurata M, Yamaguchi T, Kitagawa M, Miki Y, Yoshida K (2012) DYRK2 priming phosphorylation of c-Jun and c-Myc modulates cell cycle progression in human cancer cells. J Clin Invest 122:859-872. https://doi.org/10.1172/JCI60818

Tsuge T, Tsukaya H, Uchimiya H (1996) Two independent and polarized processes of cell elongation regulate leaf blade expansion in Arabidopsis thaliana (L.) Heynh. Development 122:1589-1600

Tsukaya H, Tsuge T, Uchimiya H (1994) The cotyledon: a superior system for studies of leaf development. Planta 195:309-312. https:// doi.org/10.1007/BF00199692

Varjosalo M, Keskitalo S, Van Drogen A, Nurkkala H, Vichalkovski A, Aebersold R, Gstaiger M (2013) The protein interaction landscape of the human CMGC kinase group. Cell Rep 3:1306-1320. https:// doi.org/10.1016/j.celrep.2013.03.027

Woods YL, Cohen P, Becker W, Jakes R, Goedert M, Wang X, Proud CG (2001) The kinase DYRK phosphorylates protein-synthesis initiation factor eIF2Bepsilon at Ser539 and the microtubuleassociated protein tau at Thr212: potential role for DYRK as a glycogen synthase kinase 3-priming kinase. Biochem J 355(Pt 3):609-615. https://doi.org/10.1042/bj3550609

Xiong Y, McCormack M, Li L, Hall Q, Xiang C, Sheen J (2013) Glucose-TOR signalling reprograms the transcriptome and activates meristems. Nature 496:181-186. https://doi.org/10.1038/natur e12030

Publisher's Note Springer Nature remains neutral with regard to jurisdictional claims in published maps and institutional affiliations. 